\title{
Potential Role of Machine Learning in Oncology
}

\author{
S Satish Kumar ${ }^{1}$, Kamran H Awan ${ }^{2}$, Shankargouda Patil ${ }^{3}$, Indu Bharkavi SK ${ }^{4}$, A Thirumal Raj ${ }^{5}$ \\ The Journal of Contemporary Dental Practice (2019): 10.5005/jp-journals-10024-2551
}

Machine learning (ML) is the ability of computers to learn from data autonomously. It is a core branch of artificial intelligence (Al), which is defined as the ability of a machine to replicate the intellectual processes of humans independently. ${ }^{1,2}$ The evolution of the microprocessor for home computers resulted in increased computing speed, efficient data collection, storage, and retrieval capacity. Thus Al techniques have evolved, which led to the discovery of artificial neural networks which are computer modeling algorithms mimicking the human brain. ${ }^{3-6} \mathrm{Al}$ has developed to an extent that it can exceed the human brain in board games such as chess, television games, image recognition, and spam e-mail filtering. In addition to engineering, arts, and finance, extensive progress has been made to integrate $\mathrm{ML}$ in the field of medicine, especially oncology.

To generate an algorithm ML uses both fed labeled data also known as supervised learning and unlabeled data also known as unsupervised learning, based on which ML formulates diagnostic, prognostic and therapeutic protocols. A prime example of ML-based application is disease risk stratification. ML-based artificial neural networks on 440 non-small cell cancer had shown $83 \%$ accuracy in risk stratification which is far superior to any conventional techniques available. ${ }^{7}$ Neural networks have also been used in pathologic diagnosis and grading of several cancers including gliomas, cervical and breast cancer. Data from ML has shown greater accuracy in predicting the survival rate among stage 1 adenocarcinoma patients than conventional tumor grading. ${ }^{8}$ Apart from diagnosis and risk assessment, ML is also used to assess the responses to treatment, and to formulate predictive models for post-therapeutic morbidity. ML has been used to generate customized treatment strategies, and to optimize therapeutic dosage in high-risk treatment modalities such as radiation therapy.

ML has also been explored in the field of drug discovery and development. Neural networks are used to predict molecular docking of a test compound into a specified biologic target. ML helps in screening for molecules more effectively and significantly reducing the cost and speed up the research process. Computational cancer biology laboratories have shown the application of $\mathrm{ML}$ derived algorithms to predict cell migration patterns in ovarian cancer. ML has also shown success in pharmacogenomics drug classification, cancer subtyping, and identification of predictive and prognostic markers. ${ }^{9,10}$ Large-scale artificial intelligence platforms like IBM Watson for oncology, integrate neural networks to process natural language, generate hypotheses, and integrate this information with medical databases to formulate customized recommendations.

Strengths of ML include processing extensive amounts of data at incredible speed, complex nonlinear relationships between variables, and update themselves as new data emerges. Despite the numerous advances, ML incurs several limitations. ML accuracy is
${ }^{1}$ Sai Srinivas Dental Care, Chennai, Tamil Nadu, India

${ }^{2}$ College of Dental Medicine, Roseman University of Health Sciences, South Jordan, USA

${ }^{3}$ College of Dentistry, Jazan University, Jazan, Saudi Arabia

${ }^{4,5}$ Sri Venkateswara Dental College and Hospital, Chennai, Tamil Nadu, India

Corresponding Author: A Thirumal Raj, Sri Venkateswara Dental College and Hospital, Chennai, Tamil Nadu, India, e-mail: thirumalraj666@gmail.com

How to cite this article: Kumar SS, Awan $\mathrm{KH}$, et al. Potential Role of Machine Learning in Oncology. J Contemp Dent Pract 2019;20(5):529-530

Source of support: Nil

Conflict of interest: None

relatively inferior in segmenting objects with poorly recognizable boundaries such as parotid glands, submandibular glands and the optic chiasma. ${ }^{11}$ Further, as machine learning algorithms get progressively more accurate in analyzing the data, results become less generalizable to the larger population. Other drawbacks include maintaining electronic health records, cost, and acceptance by medical professionals and patients. ${ }^{12}$ Though there are several limitations, the role of $\mathrm{ML}$ in oncology continues to expand. Steps should be taken to prepare our healthcare systems for the arrival of ML, which will increase research productivity and ultimately improving our ability to diagnose, prognosticate and make treatment decisions and improve patient care. ${ }^{13}$

\section{References}

1. Copeland BJ. Artificial intelligence|Definition, Examples, and Applications|Britannica.com. In: Encyclopedia Britannica. https:// www.britannica.com/technology/artificial intelligence. Accessed October 8, 2017.

2. Deo RC. Machine learning in medicine. Circulation 2015;132(20): 1920-1930. DOI: 10.1161/CIRCULATIONAHA.115.001593.

3. Majumdar B, Sarode SC, et al. Technology: artificial intelligence. $\mathrm{Br}$ Dent J 2018;224(12):916. DOI: 10.1038/sj.bdj.2018.485.

4. Aspray W. The Intel 4004 microprocessor: what constituted invention? IEEE Ann Hist Comput 1997;19(3):4-15.

5. Faggin $F$, Hoff ME, et al. The history of the 4004. IEEE Micro 1996;16(6):10-20.

6. Raj AT, Patil S, et al. Scope of mathematical biology in cancer research. J Contemp Dent Pract 2018;19(9):1035-1036.

7. Chankong T, Theera-Umpon N, et al. Automatic cervical cell segmentation and classification in Pap smears. Comput Methods Programs Biomed 2014;113(2):539-556. DOI: 10.1016/j. cmpb.2013.12.012.

8. Dey P, Logasundaram R, et al. Artificial neural network in the diagnosis of lobular carcinoma of the breast in fine-needle aspiration cytology. Diagn Cytopathol 2013;41(2):102-106. DOI: 10.1002/ dc. 21773.

(O) The Author(s). 2019Open Access This article is distributed under the terms of the Creative Commons Attribution 4.0 International License (https://creativecommons. org/licenses/by-nc/4.0/), which permits unrestricted use, distribution, and non-commercial reproduction in any medium, provided you give appropriate credit to the original author(s) and the source, provide a link to the Creative Commons license, and indicate if changes were made. The Creative Commons Public Domain Dedication waiver (http://creativecommons.org/publicdomain/zero/1.0/) applies to the data made available in this article, unless otherwise stated. 
9. Safikhani Z, Thu KL, et al. Gene isoforms as expression-based biomarkers predictive of drug response in vitro. July 2017:160937. DOI: 10.1101/160937.

10. El-Hachem N, Gendoo DMA, et al. Integrative cancer pharmacogenomics to infer large-scale drug taxonomy. Cancer Res 2017;77(11):3057-3069. DOI: 10.1158/0008-5472.CAN-17-0096.

11. Ibragimov B, Xing L. Segmentation of organs at risks in the head and neck CT images using convolutional neural networks. Med Phys
2017 Feb;44(2):547-557. http://onlinelibrary.wiley. com/doi/10.1002/ mp.12045/full. Accessed September 15, 2017.

12. Lyons JB, Koltai KS, et al. Engineering trust in complex automated systems. Ergonomics in Design: The Quarterly of Human Factors Applications 2016;24(1):13-17.

13. Khan OF, Bebb G, et al. Artificial intelligence in medicine what oncologists need to know about its potential and its limitations. Oncology Exchange 2017;16(4):8-13. 